\title{
SLP: A Zero-Contact Non-Invasive Method for Pulmonary Function Testing
}

\author{
Willem H. de Boer ${ }^{1}$ \\ wd234@cam.ac.uk \\ Joan Lasenby ${ }^{1}$ \\ jl221@cam.ac.uk \\ Jonathan Cameron ${ }^{1}$ \\ jic23@cam.ac.uk \\ Rich Wareham ${ }^{1}$ \\ rjw57@cam.ac.uk \\ Shiraz Ahmad ${ }^{1}$ \\ sa416@cam.ac.uk \\ Charlotte Roach ${ }^{1}$ \\ cr380@cam.ac.uk \\ Ward Hills ${ }^{2}$ \\ ward.hills@pneumacare.com \\ Richard Iles ${ }^{3}$ \\ richard.iles@addenbrookes.nhs.uk
}

${ }^{1}$ Department of Engineering, University of Cambridge, Cambridge

2 Pneumacare Limited, Duxford

${ }^{3}$ Addenbrookes NHS Hospital, Cambridge

\begin{abstract}
Structured Light Plethysmography (SLP) is a novel non-invasive method that uses structured light to perform pulmonary function testing that does not require physical contact with a patient. The technique produces an estimate of chest wall volume changes over time. A patient is observed continuously by two cameras and a known pattern of light (i.e. structured light) is projected onto the chest using an off-the-shelf projector. Corner features from the projected light pattern are extracted, tracked and brought into correspondence for both camera views over successive frames. A novel self calibration algorithm recovers the intrinsic and extrinsic camera parameters from these point correspondences. This information is used to reconstruct a surface approximation of the chest wall and several novel ideas for 'cleaning up' the reconstruction are used. The resulting volume and derived statistics (e.g. FVC, FEV) agree very well with data taken with a spirometer.
\end{abstract}

\section{Introduction and Background}

Pulmonary function testing (PFT) is the general term for a range of clinical tests that can be performed on a patient in order to assess respiratory status. In this paper we introduce a novel non-invasive technique to perform PFT, which requires zero contact with a patient. 
A device for performing pulmonary function tests may be broadly classified as belonging to one of two categories. The first category measures flow, also known as pneumotachography. The second category measures volume, which is known as plethysmography. Furthermore such a device may be categorised as being either 'invasive' or 'non-invasive'. An invasive procedure involves 'penetration of the body'; for PFT this generally involves use of a mouthpiece .

\subsection{Spirometry/Pneumotachography}

A spirometer is a device that is often used to perform pulmonary function tests. It consists of a mouthpiece connected to an instrument which measures the amount and the rate (flow) of air that is breathed in and out through the mouthpiece.

A typical pulmonary function test requires a patient to breathe as normally as possible through the mouthpiece. Flow is integrated to yield so-called tidal volume (TV). Another test, which demands some practice on behalf of the patient, measures someone's forced vital capacity (FVC), and forced expiratory volume (FEVx). FVC is the maximum amount of air that can be forcibly exhaled after a maximal inhalation. FEVx measures the amount of air that is forcibly exhaled some ' $\mathrm{x}$ ' seconds after maximal inhalation.

These tests are used by a clinician in assessing conditions such as asthma, pulmonary fibrosis, cystic fibrosis and other restrictive or obstructive disease. Pulmonary function tests also have applications in monitoring and evaluating patients in anaesthesia and intensive care environments.

\subsection{Plethysmography}

A pulmonary plethysmograph can be used to measure lung volume. One of the earliest of such devices - a so-called 'pulmo-meter' - dates back to the early 19th century. Ventilary volume was measured using water displacement in an inverted bell jar standing in water [ $[\mathbf{\square}]$. This technique is still employed today albeit without water: A whole body plethysmograph [ $[\mathbf{\nabla}]$ is a device that consists of a sealed, clear chamber the size of a small telephone booth, with a single mouthpiece. Changes in total body volume are measured indirectly through changes in air pressure.

Advances in the field of computer graphics, computer vision and image processing have led to the development of techniques that track multiple points on the body. A recent method uses an optical reflectance motion-analysis system (OR). Volume changes of the chest wall during respiration are tracked by computing the 3-D coordinates of markers placed on the rib cage and abdomen $[\mathbf{Q}, \mathbf{Q}]$. This technology, known as Opto-Electronic Plethysmography (OEP), has been extensively validated and shown to be applicable to adult COPD [ $⿴ 囗$ ], adult

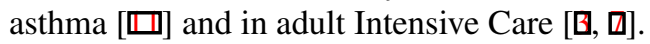

Structured light methods are a common technique used to measure 3D shape. These methods can be roughly categorised by the number of patterns that are projected onto the scene. In the simplest case a single pattern is projected [ $[\mathbb{}]$. More elaborate schemes use multiple patterns with the aim of improving reconstruction accuracy. Such approaches include binary stripe patterns $[\square]$ and phase-shifted sine wave patterns $[\square, \square]$. 


\section{Structured Light Plethysmography}

We propose a new technique called Structured Light Plethysmography. This technology is akin to OEP in that it uses computer vision and image processing methodology to estimate chest wall volume changes. In contrast to OEP, no markers need to be placed onto the body. The system has been used to monitor breathing patterns of healthy adults, sedated domestic animals and will soon be used to monitor the breathing of incubated premature babies.

SLP works by projecting a grid pattern onto a subject's chest, while in seated or supine position (see Figure 1). A grid intersection point is defined as the corner that is shared by four squares. The entire grid consists of $w_{\text {grid }} \times h_{\text {grid }}$ of these points.
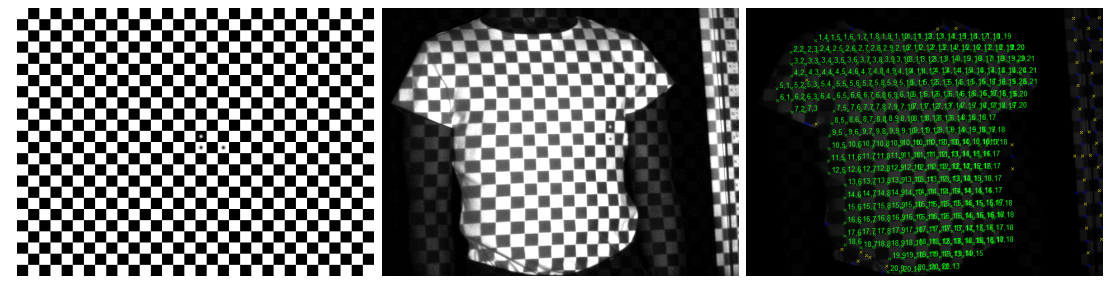

Figure 1: The grid pattern (left). The corners of the squares are 'grid intersection points' and there are $w_{\text {grid }} \times h_{\text {grid }}(23 \times 28)$ of these. The grid pattern as projected onto a subject (center), and after identifying grid intersection points (right).

Two cameras, placed at different positions and angles (both unknown), record the subject's chest area. Grid pattern intersection points are tracked, and brought into correspondence. A self-calibration algorithm then infers the internal and external parameters of the two cameras and the projector from a subset of the point correspondences. Next, the chest wall is reconstructed using the camera calibration. The reconstruction is then cleaned up to remove unwanted areas (such as limbs) and any missing points are filled in. Finally, volume changes of the chest wall over time are calculated from the reconstruction and knowledge of the work bench plane; i.e. the seat back or mat on which the subject is resting. An absolute length scale is recovered from an object of known dimensions attached to the work bench. A prototype rig was built at Cambridge University Engineering Department (CUED). This rig has been used in several (pre-)clinical tests, and is the starting point for a commercial rig currently under development [四].

\subsection{Capture Stage}

A subject is requested to rest their back firmly against the back of the seat (seated) or mat (supine) and to keep this position at all times. They are asked to wear a plain T-shirt making sure that the T-shirt fits tightly around the chest with as few creases as possible.

A researcher/clinician moves the rig in front (seated) or over (supine) the subject so that it is about 3-4 feet away. The projector is then adjusted so that the projected grid covers the entire chest area. The subject is asked to breathe in a relaxed manner for several tidal breaths. Optionally, they may be requested to perform one or more forced-expiration manoeuvres.

We define the 'work bench' as the planar surface on which the subject rests. This surface is either the back of the seat (seated) or the mat (supine). An object of known geometry is 
placed on the work bench so that we may recover the scene scale as well as the orientation of the work bench plane.

\subsection{Tracking Stage}

In order to locate potential grid intersection points, a Sobel edge detector is applied to an image frame. Non-maximal supression of the gradient magnitude of the resulting image is used to locate potential corners. Quadratic 1-D curve fitting provides sub-pixel accurate peak positioning in both directions. The user is required to approximately specify an origin in both views, once, which is used as an anchor point for finding correspondences. We map peaks to grid positions by moving outwards, starting at the origin and the 4 closest peaks, and mapping peaks to the grid by line extension from each peak in each direction. We are left with a set of grid points $\left\{\mathbf{u}_{i j}\right\}$ where $i=1,2, \ldots, w_{\text {grid }} \times h_{\text {grid }}$ and $j$ denotes the camera. We will use $\mathbf{u}_{i j}$ to denote both the invididual grid point $i$ in camera $j$ as well as the correspondence $\mathbf{u}_{i j} \leftrightarrow \mathbf{u}_{i j^{\prime}}$ where $j \neq j^{\prime}$ and $j, j^{\prime}=1, \ldots, m$. Additionally, we define $O_{i j}$ to be $O_{i j}=1$ if $\mathbf{u}_{i j}$ is known, and $O_{i j}=0$ otherwise, as not all grid points will be successfully tracked in practice. We treat the projector as a separate camera. All grid points are, trivially, known for the projector, and so $O_{i j}=1$ for all points. See Figure 1 for an example of tracked grid points in one frame.

\subsection{Calibration Stage}

We want to find both the extrinsic as well as the intrinsic parameters of the cameras. In addition, we want to infer these parameters from $\left\{\mathbf{u}_{i j}\right\}$ directly (taken over several frames spaced evenly across the duration of the measurement), a process that is called self calibration [四]. This frees us from having to perform a separate calibration step using some object of known geometry [ $[\mathbb{Q}]$, which is impractical for the kind of situations in which SLP is used. This enables us to calibrate and reconstruct the chest wall simultaneously.

We treat the cameras as pinhole cameras [四]. Given some world point $\tilde{\mathbf{X}}=(X, Y, Z, 1)$, in homogeneous coordinates, its corresponding point, $\tilde{\mathbf{x}}$, on the image plane is given by $\lambda \tilde{\mathbf{x}}=P \tilde{\mathbf{X}}$, for some $\lambda$ and $\tilde{\mathbf{x}}=(u, v, 1)$. The matrix $P$ is $3 \times 4$ and may be decomposed into the product $P=K[R \mid \mathbf{T}]$, where $R$ is a $3 \times 3$ matrix representing orientation, and $\mathbf{T}$ is a $3 \times 1$ matrix that represents translation. $K$ is of the form

$$
K=\left[\begin{array}{rrr}
f k_{u} & \gamma & u_{0} \\
0 & f k_{v} & v_{0} \\
0 & 0 & 1
\end{array}\right],
$$

with $f$ being focal length, $k_{u}, k_{v}$ being horizontal and vertical pixel scaling factors, $\gamma$ is a skew factor, and $\left(u_{0}, v_{0}\right)$ being the camera's optical centre in pixels.

A calibration technique proposed in [ㅁ] recovers camera orientation and translation for multiple cameras simultaneously from a set of point correspondences. This method optimises an objective function iteratively using closed-form formulae of the gradient of this function w.r.t. the unknown parameters. These closed-form formulae are derived using Geometric Algebra. A minimum of this objective function is assumed to correspond to an optimal calibration.

We extend the work in [प] to include the recovery of the intrinsic $K$ matrix. The extension adopts a two-stage sampling and refinement approach for the entries of $K$. While not required by the algorithm itself, but for the sake of reducing processing time, we assume 
that the skew factor $\gamma=0$ and that the optical axis $\left(u_{0}, v_{0}\right)=(0,0)$. These assumptions are entirely reasonable given the quality of today's cameras.

Each entry of $K$ must have as its units pixels. By dimensional analysis the units of $k_{u}$ and $k_{v}$ must be pixels $/ \mathrm{mm}$, assuming that $f$ is given in millimeters (mm). In fact, $k_{u}=w / w_{s}, k_{v}=$ $h / h_{s}$ where $w, h$ are the screen width and height in pixels (which are known), and $w_{s}, h_{s}$ are the camera's sensor width and height in millimeters, respectively. Expressing $h_{s}$ in terms of $w_{s}$ via the pixel aspect ratio $r=k_{v} / k_{u}$, allows us to reformulate $K$ in terms of the more intuitive unknown parameters $f, w_{s}$ and $r$ :

$$
K=\left[\begin{array}{rrr}
\frac{w}{w_{s}} f & 0 & 0 \\
0 & \frac{w}{w_{s}} r f & 0 \\
0 & 0 & 1
\end{array}\right]
$$

In the first stage of our extension we find a coarse approximation to $K$ using a simple sampling approach. In the second stage, this approximation is refined by taking the coarse approximation as a starting point for Nelder-Mead optimisation [ $\square$ ].

We require the ability to compare two different calibrations from two different $K$ matrices. Multiplication by $K$ has the effect of scaling proportionally to $f$, hence a scale difference exists between two reconstructions from different $K$ matrices [ $[$ ] . We define a comparison function that does not depend on $K$ directly, namely

$$
S_{2}^{\prime}=\sum_{j=1}^{m} \sum_{i=1}^{N}\left[\mathbf{u}_{i j}-\hat{\mathbf{u}}_{i j}\right]^{2} O_{i j},
$$

where $m$ is the number of cameras, $N$ the number of point correspondences and $\hat{\mathbf{u}}_{i j}$ is the reprojection of the $i^{\text {th }}$ reconstructed world point onto the image plane of camera $j$.

\subsubsection{Sampling}

Defining $\omega \equiv f / w_{s}$, the aim of the sampling step is to determine an initial guess for $\omega$ and $r$. We discretise that part of the solution space that we know a priori contains the true values of $\omega$ and $r$. Assume that $\omega \in\left[\omega_{\min }, \omega_{\max }\right]$ and $r \in\left[r_{\min }, r_{\max }\right] .{ }^{1}$ We discretise these intervals into sets $\Omega=\left\{\omega_{1}, \omega_{2}, \ldots, \omega_{p}\right\}$ and $R=\left\{r_{1}, r_{2}, \ldots, r_{q}\right\}$, for some suitable natural numbers $p, q$. Then, for each $\omega_{s} \in \Omega,(s=1,2, \ldots, p)$ and each $r_{t} \in R,(t=1,2, \ldots, q)$, we construct $\hat{K}\left(\omega_{s}, r_{t}\right)$ using the mapping

$$
\hat{K}:(\omega, r) \longmapsto\left[\begin{array}{rrr}
w \omega & 0 & 0 \\
0 & w \omega r & 0 \\
0 & 0 & 1
\end{array}\right]
$$

and perform the calibration routine in [ $[\mathbf{\square}]$ to find the corresponding $R$ and $\mathbf{T}$, along with the value of $S_{2}^{\prime}$. We then choose as our coarse approximation the $\hat{K}\left(\omega_{s}, r_{t}\right)$ which has the minimum value for $S_{2}^{\prime}$.

${ }^{1}$ In practice, $f$ takes on one of several discrete values, and similarly for $w_{s}$ (see [ $\left.\mathbf{\square}\right]$ ), and in general $\omega \in(0,5]$. Also, $r=\frac{h}{w} \frac{w_{s}}{h_{s}} \approx 1$ in many cases. 


\subsubsection{Refinement}

The coarse approximation can be refined by considering it as a starting point for numerical optimisation. Nelder-Mead optimisation has been chosen since it assumes very little about the objective function. This objective function is a function of $\omega$ and $r$ and can be described in the following steps.

(i) Perform calibration [四] for a candidate pair $(\hat{\omega}, \hat{r})$, using $\hat{K}(\hat{\omega}, \hat{r})$ (see Eq. 4) and $\left\{\mathbf{u}_{i j}\right\}$ or some subset thereof.

(ii) Return the corresponding value of $S_{2}^{\prime}$ as the value of our objective function at $(\hat{\omega}, \hat{r})$.

In practice, convergence is reached in fewer than 20 Nelder-Mead iterations.

To provide robustness to outliers we use RANSAC during the sampling step [س]. To save processing time, rather than also use RANSAC during refinement, we use the subset of point correspondences that were used for the best coarse approximation.

\subsection{Reconstruction Stage}

The reconstruction stage takes $\left\{\mathbf{u}_{i j}\right\}, O_{i j}$ and the camera calibration parameters and reconstructs the 3-D world positions of the grid intersection points for all cameras simultaneously by performing SVD on a linear system as discussed in [प] $]$. A surface reconstruction is generated by building a quad mesh from the 3-D world positions, which is straightforward given the grid topology. In order to calculate volume we assume that each quad lies in the convex hull of its four corners.

\subsubsection{Isolating the chest area}

Inevitably, the reconstruction contains parts of the body that do not belong to the chest wall (e.g. limbs) and these points must be discarded (see Figure 2).

We propose using a simple heuristic method applied to the binary image of the reconstruction, $B=\bigcup_{t=1}^{N} B_{t}$ (where $100 \leq N \leq t_{\max }$ ), with

$$
B_{t}=\left\{\begin{array}{l|l}
z \in E & \sum_{j=1}^{m} O_{I(z) j}(t) \geq 2
\end{array}\right\},
$$

where $I(z)$ maps pixel coordinates $z$ to a corresponding index $i, O_{i j}(t)$ is simply $O_{i j}$ at frame time $t$, and $E=\left[1, w_{\text {grid }}\right] \times\left[1, h_{\text {grid }}\right] \subset \mathbb{Z}^{2}$ (ie. an image pixel for each grid point).

Intuitively, we expand a rectangle outwards, starting from the centre of the reconstructed chest area. The rectangle is grown in the $x$ and $y$-directions separately. We stop when we have covered what is assumed to be the chest area.

The whole process is embodied in Algorithm 1. As a first step, we perform image opening [ष] with a $5 \times 5$ structuring element, $S$, yielding $B^{\prime}$. We define row-occupancy $o_{r}(c)$ for a given row $r$, centred around column $c$ to be

$$
o_{r}(c)=\sum_{c^{\prime} \mid\left(c^{\prime}, r\right) \in A} \mathbf{1}_{B^{\prime}}\left(c^{\prime}, r\right) \exp \left(-\frac{1}{2} Z\left(c^{\prime}-c\right)^{2}\right),
$$


where $\mathbf{1}_{X}$ denotes the indicator function of a set $X, A$ is the minimum bounding box s.t. $B \circ S \subseteq A$, and $Z$ is a constant

$$
Z=\frac{-8 \log (\Delta \times \sqrt{2 \pi})}{w(A)^{2}}
$$

where $\Delta$ is some small value, typically $\Delta \leq 0.001$, and $w(A)$ denotes the width of $A$ in pixels. The column-occupancy of a column, $o_{c}(r)$ for a given column $c$, centred around row $r$ is defined similarly as

$$
o_{c}(r)=\sum_{r^{\prime} \mid\left(c, r^{\prime}\right) \in A} \mathbf{1}_{B^{\prime}}\left(c, r^{\prime}\right) \exp \left(-\frac{1}{2} Z\left(r^{\prime}-r\right)^{2}\right)
$$

but now the denominator in $Z$ is $h(A)^{2}$ rather than $w(A)^{2}$, where $h(A)$ is the height of $A$ in pixels.

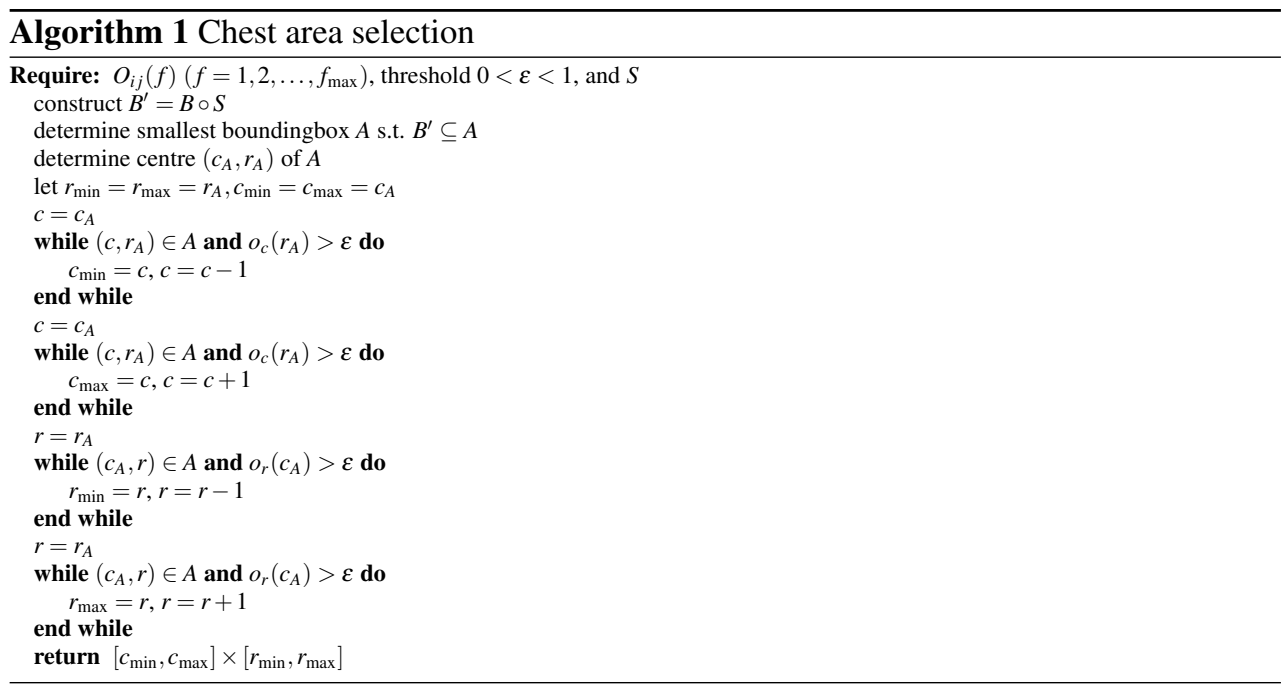

\subsubsection{Filling in missing points}

In order to faithfully calculate volume, we must fill in any missing (i.e. untracked) points (see Figure 2). We estimate such a missing point using both temporal as well as spatial (i.e. neighbouring) information from the existing reconstruction. For each frame of the reconstruction, we identify the missing points and estimate these indepenently.

Consider a single frame reconstruction at frame time $t$. Let $\left\{\mathbf{y}_{t}^{(j)}\right\}(j=1,2, \ldots, M)$ denote all $M$ successfully reconstructed (i.e. known) points at time $t$, and let $\left\{\mathbf{x}_{t}^{(i)}\right\}(i=1,2, \ldots, L)$ denote all the missing points at time $t$.

We are interested in finding an estimate for a missing point, $\hat{\mathbf{x}}_{t}^{(i)}$. Note that we are only interested in filling in points that fall within the chest area (see the previous subsection). Let $d(\mathbf{x}, \mathbf{y})$ denote the Euclidean distance between two world points $\mathbf{x}$ and $\mathbf{y}$. Suppose the known 


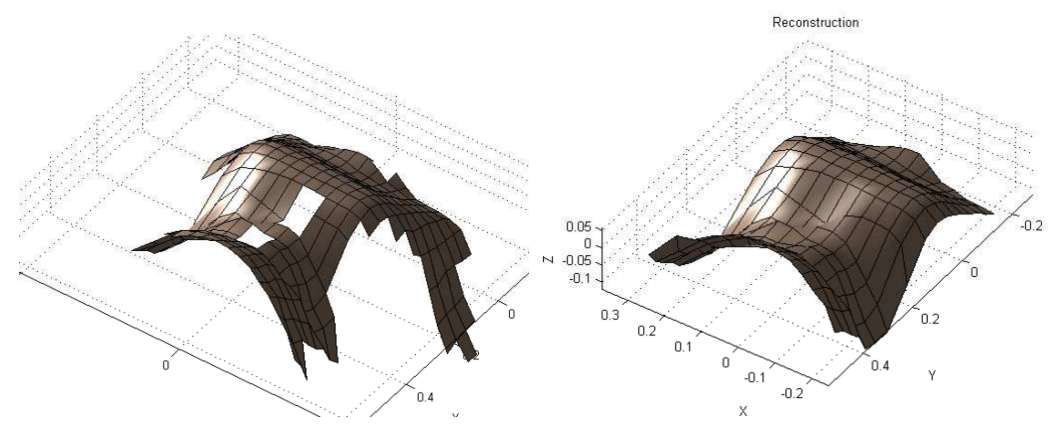

Figure 2: A reconstruction before (left) and after (right) isolating the chest area and filling in missing points.

points along row $r$ are $\mathbf{y}_{t}^{\left(I\left(i_{1}, r\right)\right)}, \mathbf{y}_{t}^{\left(I\left(i_{2}, r\right)\right)}, \ldots, \mathbf{y}_{t}^{\left(I\left(i_{n}, r\right)\right)}$ for some $n$, and $i_{1}<i_{2}<\cdots<i_{n}$. Then define $\mathbf{S}_{r}:\left[0, w_{\text {grid }}\right) \rightarrow \mathbb{R}^{3}$ to be the cubic Lagrange interpolating polynomial that minimises

$$
\sum_{k=1}^{n} d\left(\mathbf{S}_{r}\left(i_{k}-1\right), \mathbf{y}_{t}^{\left(I\left(i_{k}, r\right)\right)}\right)^{2}
$$

We define $\mathbf{S}_{c}:\left[0, h_{\text {grid }}\right) \rightarrow \mathbb{R}^{3}$ identically, but for points along column $c$. Then let

$$
\hat{\mathbf{x}}_{t}^{(i)}=\lambda_{1} \mathbf{z}_{t-1}^{(i)}+\lambda_{2} \mathbf{S}_{r}(c-1)+\lambda_{3} \mathbf{S}_{c}(r-1),
$$

where $\mathbf{z}_{t-j}^{(i)}$ is either $\mathbf{y}_{t-j}^{(i)}$ or $\hat{\mathbf{x}}_{t-j}^{(i)}$ depending on whether the point was known or estimated at time $t-j$. We impose the additional constraint that $\hat{\mathbf{x}}_{t}^{(i)}$ must lie on the line, $l$, through the projector's optical centre and the corresponding grid point on the projector's focal plane. Let $\mathbf{l}(t): \mathbb{R} \rightarrow \mathbb{R}^{3}$ denote a parametrisation of $l$. Then, the estimate is equal to the point on $\mathbf{l}(t)$ that minimises $d\left(\mathbf{l}(t), \hat{\mathbf{x}}_{t}^{(i)}\right)$.

The weight factors $\lambda_{1}, \lambda_{2}$, and $\lambda_{3}$ must satisfy the convexity constraint $\lambda_{1}+\lambda_{2}+\lambda_{3}=1$. We define a pixel 'distance' function along a row

$$
d_{r}(c)=\left\{\begin{array}{cl}
0 & \text { if } i_{1} \leq c \leq i_{n} \\
\min \left(\left|i_{1}-c\right|,\left|c-i_{n}\right|\right) & \text { otherwise }
\end{array}\right.
$$

We define pixel distance along a column, $d_{c}(r)$ identically. Then we set the spatial factors to be $\lambda_{2}=\frac{1}{\left(d_{r}(c)+1\right)^{\alpha}}, \quad \lambda_{3}=\frac{1}{\left(d_{c}(r)+1\right)^{\alpha}}$ where $\alpha \in \mathbb{R}^{+}$(typically $\alpha=1,2$ ). The temporal factor is given by $\lambda_{1}=1-\max \left\{\lambda_{2}, \lambda_{3}\right\}$. Finally, we divide by $\sum_{j} \lambda_{j}$ to satisfy the convexity constraint.

\subsection{Volume calculation Stage}

We estimate chest wall volume by calculating the volume between the reconstructed chest area and the work bench. For sake of simplicity we rotate the reconstruction such that the work bench coincides with the $z=0$ plane. The volume that we calculate is illustrated in Figure 3. 

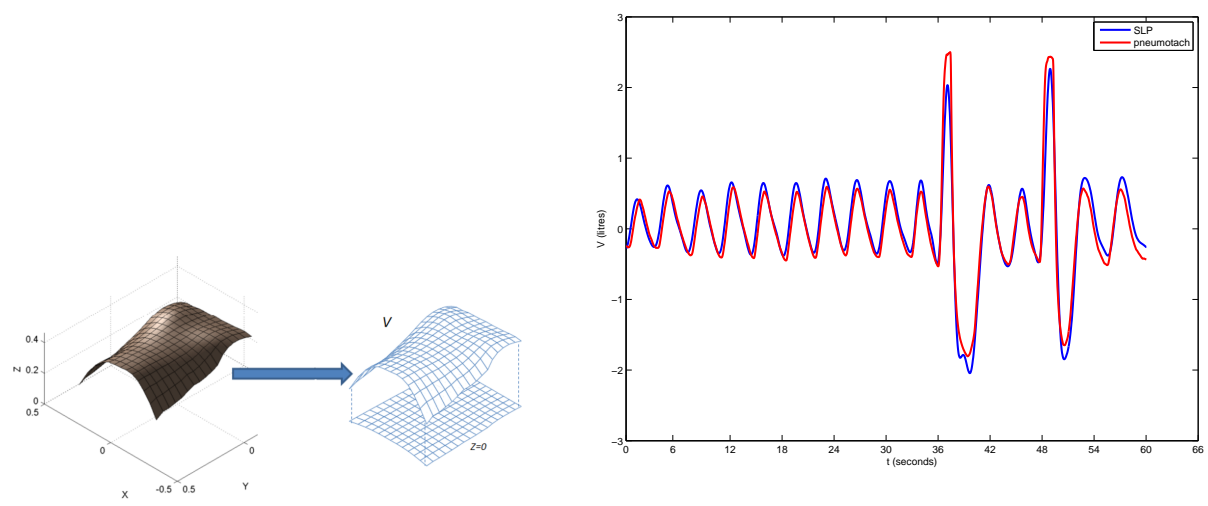

Figure 3: The 'chest wall volume' $V$ as calculated by SLP (left) and a typical example of SLP volume data superimposed on pneumotach volume data of the same measurement.

Using Gauss's Theorem, for some vector field $\mathbf{F}$ with $\nabla \cdot \mathbf{F}=1, V$ can be calculated by integrating over its boundary, $\partial V$, viz.

$$
V=\int_{\partial V} \mathbf{F} \cdot \mathbf{n} d S \equiv S_{\text {chest }}+S_{\text {side }}+S_{\text {bench }},
$$

where $S_{\text {chest }}, S_{\text {side }}, S_{\text {bench }}$ are the separate surface integrals over the chest area, the sides and the work bench, respectively. If we choose $\mathbf{F}:(x, y, z) \mapsto(0,0, z)$ then $S_{\text {side }}$ and $S_{\text {bench }}$ vanish.

Consider the quad mesh associated with a reconstruction as a set of quadrilaterals, $\mathbf{Q}=$ $\left\{Q_{j}\right\}\left(j=1,2, \ldots, N_{Q}\right.$, with $N_{Q}$ being the total number of quadrilaterals). We can triangulate a given $Q_{j}$ in two different ways. Suppose $\mathbf{T}=\left\{T_{i}\right\}\left(i=1,2, \ldots, 2 N_{Q}\right)$ is an arbitrary triangulation of $\mathbf{Q}$. Then $S_{\text {chest }}=\sum_{i=1}^{2 N_{Q}} \int_{T_{i}} \mathbf{F} \cdot \mathbf{n} d S$, or $S_{\text {chest }}$ is the sum of the integrals over the triangles in $\mathbf{T}$. Now let $\mathbf{p}_{0}, \mathbf{p}_{1}, \mathbf{p}_{2}$ be the vertices of triangle $T_{i}$ defined in an anti-clockwise direction. Using elementary calculus we find the closed-form solution

$$
\int_{T_{i}} \mathbf{F} \cdot \mathbf{n} d S=\left(\mathbf{r}_{1} \times \mathbf{r}_{2}\right) \cdot\left[\left(\frac{\mathbf{p}_{0}}{2}+\frac{\mathbf{r}_{1}+\mathbf{r}_{2}}{6}\right) \cdot \mathbf{k}\right] \mathbf{k},
$$

where $\mathbf{r}_{1}=\mathbf{p}_{1}-\mathbf{p}_{0}$ and $\mathbf{r}_{2}=\mathbf{p}_{2}-\mathbf{p}_{0}$, and $\mathbf{a} \times \mathbf{b}$ denotes the vector cross-product between vectors $\mathbf{a}$ and $\mathbf{b}$. The two possible triangulations of a quadrilateral $Q_{j}$ result in two integrals: $i_{Q_{j}}$, and $I_{Q_{j}}$, say, and $i_{Q_{j}} \leq I_{Q_{j}}$ (with equality if the corners of $Q_{j}$ are co-planar). The convex hull of $Q_{j}$ is the tetrahedron bounded by the two triangulations of $Q_{j}$. Hence letting $s \equiv \sum_{j} i_{Q_{j}}$ and $S \equiv \sum_{j} I_{Q_{j}}$ we have $s \leq S_{\text {chest }} \leq S$. In practice we assume $S_{\text {chest }} \approx \frac{s+S}{2}$ to be a good approximation therefore an estimate of volume is taken as $\hat{V}=\frac{s+S}{2}$.

\section{Experiments}

To validate the SLP system we used a laptop-based CardinalHealth UK MasterScope spirometer. This system outputs a flow signal which can be exported in a machine-readable format, 
and integrated to yield volume. At the start of each test session, the values for the ambient conditions were fed into the spirometer and the system was calibrated using a 1 litre syringe.

SLP measurements were performed on 40 healthy subjects (male/female, age $27 \pm 12$ years, height $176 \pm 5.6 \mathrm{~cm}$, weight $74 \pm 14 \mathrm{~kg}$ ). The subject was asked to wear a nose clip, hold the spirometer head in the left hand, and breathe in relaxed manner through the filtermouthpiece attachment and perform one or two forced expirations.

SLP measures chest wall volume changes whereas pneumotach infers lung volume. In order to make a meaningful comparison the SLP data and pneumotach data were normalised. A scale factor was applied to the SLP volume data so as to agree with the range of tidal breathing volume of the pneumotach. The SLP volume data correlate very well $\left(R^{2}=0.91\right)$ with that of a pneumotach. Forced expiration data also correlate well $\left(R^{2}=0.97\right)$.

In order to quantify the accuracy of the SLP volume we calculated the volume swept out by one square (see Figure 1) as projected onto a flat plate as it is moved towards the rig over a distance that is typical during an actual measurement with a standard system setup. The mean SLP volume calculated for a sample of 100 instances was within $0.7 \%$ of the true volume, taking projector foreshortening into account. More extensive evaluations are currently being performed.

\section{Conclusion}

We have proposed a new technique for performing PFT that requires no physical contact with a patient. Estimates of chest wall volume changes over time and its derived statistics (FEV, FVC, etc) correlate very well with those produced with a spirometer.

The prototype rig is now being used for performing clinical trials and more extensive validation tests in cooperation with Addenbrookes NHS Hospital, Cambridge.

The current algorithm would fail if the patient moves away from the work bench in any way. Future work may include fitting a model of the chest to the reconstruction to combat this problem. We are also experimenting with replacing the single grid pattern with multiple phase-shifted sine wave paterns.

\section{References}

[1] Pneumacare Ltd. http: / / www . pneumacare. com.

[2] Standard sensor sizes. http://www.dpreview.com/learn/?/key= Sensor_sizes.

[3] A. Aliverti, R. L. Dellaca, R. Pelosi, D. Chiumello, A. Pedotti, and L. Gatinoni. Optoelectronic plethysmography in intensive care patients. Am J Resp Critic Care Med, 161:1546-1552, 2000.

[4] A. Aliverti, R. Dellaca, N. Stevenson, A. Pedotti, A. Lo Mauro, and P. M. A. Calverley. Effects of bronchodilators on subdivisions of lung and chest wall volume in copd. Eur Respir J, 20(38):13, 2002.

[5] S. J. Cala, C. Kenyon, G. Ferrigno, P. Carnevali, A. Aliverti, A. Pedotti, P. T. Macklem, and D. F. Rochester. Chest wall and lung volume estimation by optical reflectance motion analysis. J Appl Physiol, 81:2680-2689, 1996. 
[6] W. H. de Boer. Structured Light Plethysmography: A non-invasive method for pulmonary function testing using visible light. MPhil thesis, University of Cambridge, Dep. of Engineering, 2010.

[7] R. L. Dellaca, A. Aliverti, P. Pelosi, E. Carlesso, D. Chiumello, and A. Pedotti. Estimation of end-expiratory lung volume variations by optoelectronic plethysmography. Crit Care Med, 29(9):1807-1811, 2001.

[8] A. B. DuBois, S. Y. Botelho, and J. H. Comroe Jr. A new method for measuring airway resistance in man using a body plethysmograph: Values in normal subjects and in patients with respiratory disease. Journal of Clin. Invest., pages 327-335, 1956.

[9] G. Ferrigno, P. Carnevali, A. Aliverti, F. Molteni, G. Beulcke, and A. Pedotti. Threedimensional optical analysis of chest wall motion. J Appl Physiol, 77:1224-1231, 1994.

[10] M. A. Fischler and R. C. Bolles. Random sample consensus: A paradigm for model fitting with applications to image analysis and augomated cartography. Communications of the ACM, 24:183-395, 1981.

[11] M. Gorini, I. Iandelli, G. Misuri, F. Bertoli, M. Filippelli, M. Mancini, R. Duranti, F. Gigliotti, and G. Scano. Chest wall hyperinflation during acute bronchoconstriction in asthma. Am J Resp Critic Care Med, 160:808-816, 1999.

[12] R. I. Hartley and A. Zisserman. Multiple View Geometry in Computer Vision. Cambridge University Press, ISBN: 0521540518, second edition, 2004.

[13] E. Kentish. An account of baths and of a madeira-house at bristol: With a drawing and description of a pulmometer and cases shewing its utility in ascertaining the state of the lungs in disease of the chest. Longman, Hurst, Rees, Orme and Browne, 1814.

[14] J. Lasenby and A. Stevenson. Geometric Algebra with Applications in Science and Engineering, chapter 8, pages 147-169. Birkhauser, ISBN: 0817641998, 2001.

[15] J. A. Nelder and R. Mead. A simplex method for function minimization. Computer Journal, 7:308-313, 1965.

[16] S. Rusinkiewicz, O. Hall-Holt, and M. Levoy. Real-time 3d model acquisition. ACM Transactions on Graphics (Proceedings of ACM SIGGRAPH 2002), 21(3):438-446, 2002.

[17] D. Scharstein and R. Szeliski. High-accuracy stereo depth maps using structured light. In IEEE Comp. Soc. Conf. on Computer Vision and Pattern Recognition, volume 1, pages 195-202, 2003.

[18] L. G. Shapiro and G. C. Stockman. Computer Vision. Prentice Hall, Inc., 1 edition, 2001.

[19] T. Svoboda, D. Martinec, and T. Pajdla. A convenient multi-camera self-calibration for virtual environments. In PRESENCE: Teleoperators and Virtual Environments, volume 14, 2005. 
[20] R. Y. Tsai. An efficient and accurate camera calibration technique for $3 \mathrm{~d}$ machine vision. In Proceedings of IEEE Conference on Computer Vision and Pattern Recognition (CVPR), pages 364-374, 1986.

[21] L. Zhang, B. Curless, and S. M. Seitz. Rapid shape acquisition using color structured light and multi-pass dynamic programming. In The 1st IEEE Int. SYmp. on 3D Data Processing, Visualization, and Transmission, pages 24-36, 2002.

[22] S. Zhang and P. S. Huang. High-resolution, real-time three-dimensional shape measurement. In Optical Engineering, volume 45, 2006. 